\title{
Well-Posedness for Set Optimization Problems ${ }^{1}$
}

\author{
W. Y. ZHANG AND S. J. LI ${ }^{2}$ \\ College of Mathematics and Science, \\ Chongqing University, Chongqing, 400044, China \\ E-mails: wenyanzhang@126.com and lisj@cqu.edu.cn \\ K. L. TEO \\ Department of Mathematics and Statistics, \\ Curtin University of Technology, G.P.O. Box U1987, Perth, WA 6845, Australia \\ E-mail: K.L.Teo@curtin.edu.au
}

February 5, 2009

\begin{abstract}
In this paper, three kinds of well-posedness for set optimization are first introduced. By virtue of a generalized Gerstewitz's function, the equivalent relations between the three kinds of well-posedness and the well-posedness of three kinds of scalar optimization problems are established, respectively. Then, sufficient and necessary conditions of well-posedness for set optimization problems are obtained by using a generalized forcing function, respectively. Finally, various criteria and characterizations of well-posedness are given for set optimization problems.
\end{abstract}

Keywords: Well-posedness; Set optimization; Gerstewitz's function.

\footnotetext{
${ }^{1}$ This research was partially supported by the National Natural Science Foundation of China (Grant numbers: 10871216 and 60574073).

${ }^{2}$ Corresponding author.
} 


\section{Introduction}

Well-posedness of optimization problems was first studied by Tykhonov [1] in 1966. Since then, the notions of well-posedness have been extended to different kinds of optimization problems (see $[2,3,4,5,6,7]$ ). In the book edited by Lucchetti and Revalski [8], Loridan gave a survey on some theoretical results of well-posedness, approximate solutions and variational principles in vector optimization. Based on the $\varepsilon$-minimal solutions, Bednarczuk [9] investigated several well-posedness for vector optimization problems. Huang [2] introduced two kinds of extended well-posedness for set-valued optimization problems and investigated a series of their characterizations and criteria. Also, some authors discussed the well-posedness of variational inequality and equilibrium problems, see [7, 10, 11].

It is worth noting that there is a kind of optimization problems called set optimization problems, which was firstly introduced by Kuroiwa (see [12]). Comparing with the usual set-valued optimization problems, set optimization problems consider relationship among image sets, but not look for efficient points of the set of all image sets. Thus, set optimization problems often play more natural roles. Until now there have been many papers to study them (see $[12,13,14,15,16,17,18]$ ). Kuroiwa [13] showed six relations among sets, and obtained duality theorems of set optimization. Kuroiwa [15] introduced efficiencies for a family of sets and investigated existence results of such efficient sets. Using the concept of cone extension and the Mordukhovich coderivative, Ha [16] studied some variants of the Ekeland's variational principle for a set-valued mapping under various continuity assumptions. Alonso and Rodríguez-Marín [18] discussed the optimality conditions for set optimization. But to the best of our knowledge, there is still no paper concerning well-posedness for set optimization problems.

In this paper, we shall first introduce three kinds of well-posedness for a set optimization problem, i.e., $k_{0}$-well-posedness at a minimizer, generalized $k_{0}$-well-posedness and extended $k_{0}$-well-posedness. Then, using a generalized version of so-called nonlinear scalarization functional (see [17]), we establish equivalent relations between the three kinds of well-posedness for the set optimization problem and well-posedness of the three kinds of scalar optimization problems, respectively. Finally, base on these scalar results, we extend some basic results of well-posedness of scalar optimization problems to set optimization problems and derive some criteria and characterizations for the three types of well-posedness of the set optimization problem.

The rest of the paper is organized as follows. In Section 2, we present the concepts 
of three kinds of well-posedness for a set optimization problem and give examples to illustrate them. In Section 3, we prove the equivalent relations between three kinds of wellposedness of the set optimization problem and well-posedness of the three kinds of scalar optimization problems, respectively, and extend many basic results of scalar optimization problems to the set optimization problem. In Section 4, we give some characterizations and criteria to the three kinds of well-posedness for the set optimization problem.

\section{Preliminaries and Well-Posedness of $(X, I)$}

Let $(X, d)$ be a metric space, and $Y$ be a real topological linear space ordered by a convex closed and pointed cone $C \subset Y$ with its topological interior $\operatorname{int} C \neq \emptyset$. Let $k_{0} \in \operatorname{int} C$ and $e=-k_{0}$. We say that a nonempty set $A \subset Y$ is $C$-proper if $A+C \neq Y$ and denote by $\mathcal{P}_{0 C}(Y)$ the family of all $C$-proper subsets of $Y . A \subset Y$ is said to be $C$-closed if $A+C$ is a closed set, $C$-bounded if for each neighborhood $U$ of zero in $Y$ there is some positive number $t$ such that $A \subset t U+C$ and $C$-compact if any cover of $A$ of the form $\left\{U_{\alpha}+C: U_{\alpha}\right.$ are open $\}$ admits a finite subcover. $A \subset Y$ is said to be bounded if for each neighborhood $U$ of zero in $Y$ there is some positive number $t$ such that $A \subset t U$. Suppose that $A, B \subset Y$. By $A \leq_{C} B$ and $A \leq_{\text {int } C} B$ we denote $B \subset A+C$ and $B \subset A+\operatorname{int} C$, respectively. Similarly, by $A \not_{C} B$ and $A \not_{\text {int } C} B$ we denote $B \not \subset A+C$ and $B \not \subset A+\operatorname{int} C$, respectively.

Assume that $I: X \rightarrow 2^{Y}$ is a set-valued mapping with nonempty values at each point in $X$. $I$ is said to be bounded (closed, compact, convex, $C$-closed, $C$-bounded, $C$ compact)-valued if for each $x \in X, I(x)$ is a bounded (closed, compact, convex, $C$-closed, $C$-bounded, $C$-compact) set. $I$ is said to be $C$-lower semi-continuous iff, for any $A \subset Y$, the set $\left\{x \in X: I(x) \leq_{C} A\right\}$ is closed. $I$ is said to be upper semi-continuous (u.s.c. for short) at $x \in X$ if for any open set $U \supset I(x)$, there exists a neighborhood $V$ of $x$ such that $\bigcup_{x \in V} I(x):=I(V) \subset U$. I is said to be u.s.c. on $X$ if $I$ is u.s.c. at every point of $X . I$ is said to be lower semi-continuous (l.s.c. for short) at $x^{0} \in X$ if for any $y^{0} \in I\left(x^{0}\right)$ and any neighborhood $U$ of $y^{0}$, there exists a neighborhood $V$ of $x^{0}$ such that $\forall x \in V$, $I(x) \cap U \neq \emptyset$. $I$ is said to be l.s.c. on $X$ if $I$ is l.s.c. at every point of $X$. $I$ is said to be continuous, if $I$ is both l.s.c. and u.s.c. on $X$.

Consider the following set optimization problem:

$$
(X, I): \text { minimize } I(x), x \in X \text {. }
$$


A point $y \in X$ is said to be a minimizer of $(X, I)$ if and only if $\forall x \in X, I(x) \leq_{C} I(y)$ implies $I(y) \leq_{C} I(x)$, and the set of all minimizers is denoted by $\operatorname{argmin}(X, I)$. A point $y \in X$ is said to be a weak minimizer of $(X, I)$ if and only if $I(x) \mathbb{L}_{\text {int } C} I(y)$, for all $x \in X$, and the set of all weak minimizers is denoted by $\operatorname{argwmin}(X, I)$. Clearly, a minimizer of $(X, I)$ must be a weak minimizer of $(X, I)$, but the reverse may not hold. In this paper, we always assume that $\operatorname{argmin}(X, I) \neq \emptyset$ and $\operatorname{argwmin}(X, I) \neq \emptyset$.

Now we introduce three kinds of well-posedness for set optimization problem $(X, I)$.

Definition $2.1(X, I)$ is said to be $k_{0}$-well-posed at $v \in \operatorname{argmin}(X, I)$, if for each sequence $\left\{x_{n}\right\}$, which satisfies that $\exists \varepsilon_{n}>0, \varepsilon_{n} \rightarrow 0$ such that

$$
I\left(x_{n}\right) \leq_{C} I(v)+\varepsilon_{n} k_{0},
$$

it holds that $x_{n} \rightarrow v$.

The sequence $\left\{x_{n}\right\}$ as in (1) is called a $k_{0}$-minimizing sequence to $v \in \operatorname{argmin}(X, I)$.

Well-posedness defined in Definition 2.1 is a notion to study the behavior of the function at one point in $\operatorname{argmin}(X, I)$. The following two definitions investigate the behavior of the variables when the corresponding objective function values are approached in different means to the sets of minimizers.

Definition $2.2(X, I)$ is said to be generalized $k_{0}$-well-posed, if for each sequence $\left\{x_{n}\right\}$, which satisfies that $\exists \varepsilon_{n}>0, \varepsilon_{n} \rightarrow 0$ and $\exists z_{n} \in \operatorname{argmin}(X, I)$ such that

$$
I\left(x_{n}\right) \leq_{C} I\left(z_{n}\right)+\varepsilon_{n} k_{0}
$$

there exist a subsequence $\left\{x_{n_{k}}\right\} \subset\left\{x_{n}\right\}$ and $x^{*} \in \operatorname{argmin}(X, I)$ such that $x_{n_{k}} \rightarrow x^{*}$.

The sequence $\left\{x_{n}\right\}$ as in (2) is called a generalized $k_{0}$-minimizing sequence.

Definition $2.3(X, I)$ is said to be extended $k_{0}$-well-posed, if for every sequence $\left\{x_{n}\right\}$, which satisfies that $\exists \varepsilon_{n}>0, \varepsilon_{n} \rightarrow 0, \forall x \in X$,

$$
I\left(x_{n}\right) \not \subset I(x)+\varepsilon_{n} k_{0}+i n t C,
$$

there exist a subsequence $\left\{x_{n_{k}}\right\} \subset\left\{x_{n}\right\}$ and $x^{*} \in \operatorname{argwmin}(X, I)$ such that $x_{n_{k}} \rightarrow x^{*}$.

The sequence $\left\{x_{n}\right\}$ as in (3) is called an extended $k_{0}$-minimizing sequence. 
If problem $(X, I)$ is neither generalized $k_{0}$-well-posed nor extended $k_{0}$-well-posed, then it is called $k_{0}$-ill-posed.

Let us illustrate these definitions by the following examples.

Example 2.1 Let $X=R, C=R_{+}^{2}$ and $k_{0}=(1,1)$.

(i) Let the mapping $F_{1}:[0,4 \pi] \rightarrow R^{2}$ be defined by

$$
F_{1}(x)=\left\{\begin{array}{l}
(x, \sin x)+\left\{\left(y_{1}, y_{2}\right) \mid y_{1}^{2}+y_{2}^{2}=\frac{1}{9}\right\}, \text { if } x \in[0,2 \pi), \\
(x, \sin x)+\left\{\left(y_{1}, y_{2}\right) \mid y_{1}^{2}+y_{2}^{2}=\frac{1}{4}\right\}, \text { if } x \in[2 \pi, 4 \pi] .
\end{array}\right.
$$

Then, set optimization problem $\left(X, F_{1}\right)$ is $k_{0}$-well-posed at $x=\frac{3 \pi}{2}$ and $x=\frac{7 \pi}{2}$.

(ii) Let the mapping $F_{2}:[0,+\infty) \rightarrow R^{2}$ be defined by

$$
F_{2}(x)=\{\lambda(0,1)+(1-\lambda)(x, 0): 0 \leq \lambda \leq 1\} .
$$

Then set optimization problem $\left(X, F_{2}\right)$ is generalized $k_{0}$-well-posed.

(iii) Let the mapping $F_{3}:(0,+\infty) \rightarrow R^{2}$ be defined by

$$
F_{3}(x)=(x-n, n)+[0,1] \times[0,1], x \in(n, n+1], n=0,1, \ldots
$$

Then, set optimization problem $\left(X, F_{3}\right)$ is extended $k_{0}$-well-posed.

(iv) Let the mapping $F_{4}:(-\infty, 0] \rightarrow R^{2}$ be defined by

$$
F_{4}(x)=\left\{\begin{array}{l}
\{(0, u), 0 \leq u \leq 1\}, \text { if } x=0, \\
\left\{\left(2^{x}, u\right), 0 \leq u \leq \frac{1}{1-2^{x}}\right\}, \text { if } x \in(-\infty, 0) .
\end{array}\right.
$$

Then, set optimization problem $\left(X, F_{4}\right)$ is $k_{0}$-ill-posed.

Remark 2.1 (1) It is not difficult to see that $k_{0}$-minimizing sequence to $v \in \operatorname{argmin}(X, I)$ and generalized $k_{0}$-minimizing sequence are always exist. From Proposition 3.1 of [16], when $I(X)$ is $C$-bounded, extended $k_{0}$-minimizing sequence exists.

(2) Suppose that $(X, I)$ is $k_{0}$-well-posed at $v \in \operatorname{argmin}(X, I)$, then there exists no point $u \in X, u \neq v$ satisfying both $I(v) \leq_{C} I(u)$ and $I(u) \leq_{C} I(v)$.

(3) Problem $(X, I)$ is generalized $k_{0}$-well-posed iff $\operatorname{argmin}(X, I)$ is compact and $d\left(x_{n}\right.$, $\operatorname{argmin}(X, I)) \rightarrow 0$ for every generalized $k_{0}$-minimizing sequence $\left\{x_{n}\right\} .(X, I)$ is extended $k_{0}$-well-posed iff $\operatorname{argwmin}(X, I)$ is compact and $d\left(x_{n}, \operatorname{argwmin}(X, I)\right) \rightarrow 0$ for every extended $k_{0}$-minimizing sequence $\left\{x_{n}\right\}$. 
(4) Assume that $\operatorname{argmin}(X, I)$ is compact and $I$ is continuous. If problem $(X, I)$ is $k_{0}$ well-posed at $y$ for each $y \in \operatorname{argmin}(X, I)$, then $(X, I)$ is generalized $k_{0}$-well-posed.

Next we recall the definitions of well-posedness and and generalized well-posedness for a scalar optimization problem in [19]. Let $f: X \rightarrow R$ be a real-valued function. Consider the following scalar optimization problem:

$$
(X, f): \min _{x \in X} f(x) .
$$

- $(X, f)$ is called Tykhonov well-posed iff $f$ has an unique minimizer on $X$ towards which every sequence $u_{n} \in X$ such that $f\left(u_{n}\right) \rightarrow \inf f(X)$ converges.

- $(X, f)$ is called generalized well-posed in the scalar sense iff the set of minimizers of $(X, f)$ is not empty, and every sequence $\left\{u_{n}\right\} \subset X$ such that $f\left(u_{n}\right) \rightarrow \inf f(X)$ has some subsequence $\left\{u_{n_{k}}\right\}$ converging to a minimizer of $(X, f)$.

Remark 2.2 If $Y=R, C=R_{+}, k_{0}=1$ and $I$ is single-valued, then $k_{0}$-well-posed at $v \in \operatorname{argmin}(X, I)$ reduces to the Tykhonov well-posedness in [19]. The generalized $k_{0}$-well-posedness and extended $k_{0}$-well-posedness for $(X, I)$ reduce to the generalized wellposedness in the scalar sense (see [19]).

\section{Scalarization and Well-posedness of $(X, I)$}

In this section, we recall the Gerstewizt's function studied in [20] and discuss the equivalent relations of three kinds of well-posedness between set optimization problems and scalar optimization problems, respectively.

Definition 3.1 ([20]) Let $a \in Y$. It is said that $\phi_{e, a}: Y \rightarrow R$ defined by

$$
\phi_{e, a}(y)=\min \{t \in R: y \in t e+a+C\}, \text { for } y \in Y,
$$

is the Gerstewizt's function.

The Gerstewizt's function is continuous and strictly decreasing on $Y$. This function is also called nonlinear scalarization functional. It plays important roles in many areas.

Based on Definition 3.1, Hernández and Rodríguez-Marín (see [17]) introduced a generalized Gerstewitz's function as follows. 
Definition 3.2 ([17]) Let the function $G_{e}(\cdot, \cdot): \mathcal{P}_{0 C}(Y)^{2} \rightarrow R \cup\{\infty\}$ defined by setting

$$
G_{e}(A, B)=\sup _{b \in B}\left\{\phi_{e, A}(b)\right\}, \text { for }(A, B) \in \mathcal{P}_{0 C}(Y)^{2}
$$

where the function $\phi_{e, A}: Y \rightarrow R \cup\{-\infty\}$ is defined by

$$
\phi_{e, A}(y)=\inf \{t \in R: y \in t e+A+C\}, \text { for } y \in Y \text {. }
$$

Note that when $A=\{a\}$ and $B=\{y\}$, the function $G_{e}(A, B)$ reduces to the function $\phi_{e, a}(y)$.

From Proposition 3.2, Theorem 3.6 and Theorem 3.9 of [17], we immediately obtain the following important properties of $G_{e}(\cdot, \cdot)$.

Lemma 3.1 ([17]) Let $A$ be a $C$-bounded set and $B \in \mathcal{P}_{0 C}(Y)$. Then $B$ is $C$-bounded if and only if $G_{e}(A, B)<\infty$.

Lemma 3.2 ([17]) $A$ is $C$-closed and $B$ is $C$-bounded. Then the following equality holds:

$$
G_{e}(A, B)=\min \{r \mid B \subset r e+A+C\}
$$

Lemma 3.3 ([17]) Assume that $r \in R, A$ is $C$-closed and $B$ is $C$-bounded. Then,

(i) $G_{e}(A, B) \leq r \Leftrightarrow B \subset r e+A+C$;

(ii) If $B_{1}$ and $B_{2}$ are $C$-compact sets and $B_{2} \leq{ }_{\text {int } C} B_{1}$, then $G_{e}\left(A, B_{1}\right)<G_{e}\left(A, B_{2}\right)$;

(iii) $G_{e}(A, A)=0$.

Lemma 3.4 Assume $A, A_{1}, A_{2}, B \in \mathcal{P}_{0 C}(Y), r \in R, A, A_{1}, A_{2}$ are $C$-closed and $B$ is C-bounded. Then,

(i) $G_{e}\left(A+\varepsilon k_{0}, B\right)=G_{e}(A, B)+\varepsilon$, for all $\varepsilon \geq 0$;

(ii) $G_{e}\left(A, B+\varepsilon k_{0}\right)=G_{e}(A, B)-\varepsilon$, for all $\varepsilon \geq 0$;

(iii) If $A_{1} \leq_{C} A_{2}$, then $G_{e}\left(A_{1}, B\right) \leq G_{e}\left(A_{2}, B\right)$;

(iv) $G_{e}(A, B)<r \Leftrightarrow B \subset r e+A+i n t C$. 
Proof. (i) From the properties of the function $G_{e}(\cdot, \cdot)$,

$$
\begin{aligned}
G_{e}\left(A+\varepsilon k_{0}, B\right) & =\min \left\{r \mid B \subset r e+A+\varepsilon k_{0}+C\right\} \\
& =\min \{r \mid B \subset(r-\varepsilon) e+A+C\} \\
& =\min \{r-\varepsilon \mid B \subset(r-\varepsilon) e+A+C\}+\varepsilon \\
& =G_{e}(A, B)+\varepsilon .
\end{aligned}
$$

(ii) From the properties of the function $G_{e}(\cdot, \cdot)$,

$$
\begin{aligned}
G_{e}\left(A, B+\varepsilon k_{0}\right) & =\min \left\{l \mid B+\varepsilon k_{0} \subset l e+A+C\right\} \\
& =\min \{l \mid B \subset(l+\varepsilon) e+A+C\} \\
& =\min \{l+\varepsilon \mid B \subset(l+\varepsilon) e+A+C\}-\varepsilon \\
& =G_{e}(A, B)-\varepsilon .
\end{aligned}
$$

(iii) Since

$$
\begin{gathered}
G_{e}\left(A_{2}, B\right)=\min \left\{r \mid B \subset r e+A_{2}+C\right\}, \\
B \subset G_{e}\left(A_{2}, B\right) e+A_{2}+C .
\end{gathered}
$$

Let $A_{1} \leq_{C} A_{2}$. Then we have

$$
B \subset G_{e}\left(A_{2}, B\right) e+A_{1}+C \text { and } G_{e}\left(A_{1}, B\right) \leq G_{e}\left(A_{2}, B\right) .
$$

(iv) Suppose $G_{e}(A, B)<r$. Then there exists an $\lambda<r$ such that $G_{e}(A, B) \leq \lambda$. It follows from Lemma 3.3 (i) that

$$
\begin{aligned}
B & \subset \lambda e+A+C \\
& =(\lambda-r+r) e+A+C \\
& =r e+A-(r-\lambda) e+C .
\end{aligned}
$$

By $-(r-\lambda) e \in \operatorname{int} C$, we have $B \subset r e+A+\operatorname{int} C$.

Conversely, if $B \subset r e+A+\operatorname{int} C$, then for every $b \in B$, there exist $a \in A$ and $c \in \operatorname{int} C$ such that $b=r e+a+c$. Since $Y$ is a linear topological space, one can find a real number $t>0$ such that $c+t e \in C$. Set $\lambda:=r-t$, we get

$$
b=r e+a+c=(t+\lambda) e+a+c=\lambda e+a+c+t e .
$$

Hence $y \in \lambda e+a+C$. This implies $B \subset \lambda e+A+C$. Thus, from Lemma 3.3 (i), we have $G_{e}(A, B) \leq \lambda<r$. The proof is complete. 
Theorem 3.1 Suppose that $v \in \operatorname{argmin}(X, I)$ and $I$ is $C$-bounded-valued and $C$-closedvalued. Then,

(i) the problem $(X, I)$ is $k_{0}$-well-posed at $v$ if and only if the problem $\left(X, G_{e}(I(\cdot), I(v))\right)$ is Tykhonov well-posed;

(ii) the problem $(X, I)$ is generalized $k_{0}$-well-posed if and only if $\operatorname{argmin}(X, I)$ is compact and the scalar problem $\left(X, \inf _{v \in \operatorname{argmin}(X, I)} G_{e}(I(\cdot), I(v))\right)$ is generalized well-posed in the scalar sense;

(iii) if $I$ is $C$-compact-valued, the problem $(X, I)$ is extended $k_{0}$-well-posed if and only if $\operatorname{argwmin}(X, I)$ is compact and the scalar problem $\left(X,-\inf _{x \in X} G_{e}(I(x), I(\cdot))\right)$ is generalized well-posed in the scalar sense.

Proof. First, we prove that for $v \in \operatorname{argmin}(X, I)$ and every $x \in X, G_{e}(I(x), I(v)) \geq 0$. On a contrary, suppose that there exist $x \in X$ and $r<0$, such that $G_{e}(I(x), I(v))<r$. From Lemma 3.4 (iv), we have

$$
I(v) \subset r e+I(x)+\operatorname{int} C \subset I(x)+\operatorname{int} C .
$$

Since $v$ is a minimizer of $(X, I), I(x) \subset I(v)+C$. Therefore, $I(x) \subset I(x)+\operatorname{int} C$, which is a contradiction.

Next, we prove that for $x \in X, G_{e}(I(x), I(v))=0$ if and only if $I(x) \leq_{C} I(v)$ and $I(v) \leq_{C} I(x)$. In fact, combining with $G_{e}(I(x), I(v))=0$ and $v \in \operatorname{argmin}(X, I)$, we have that

$$
I(v) \subset I(x)+C \text { and } I(x) \subset I(v)+C,
$$

i.e.,

$$
I(x) \leq_{C} I(v) \text { and } I(v) \leq_{C} I(x) .
$$

Conversely, assume $I(x) \subset I(v)+C$ and $I(v) \subset I(x)+C$. From $I(v) \subset I(x)+C$, we have $G_{e}(I(x), I(v)) \leq 0$. If $G_{e}(I(x), I(v))<0$, then there exists $r<0$ such that $G_{e}(I(x), I(v))<r$. From Lemma 3.4 (iv), we have $I(v) \subset r e+I(x)+\operatorname{int} C$. Thus,

$$
I(x) \subset I(v)+C \subset r e+I(x)+\operatorname{int} C \subset I(x)+\operatorname{int} C,
$$

which is a contradiction.

Finally, we prove that (i), (ii) and (iii) hold, respectively. 
(i) Assume that $\left\{x_{n}\right\}$ is a sequence satisfying

$$
G_{e}\left(I\left(x_{n}\right), I(v)\right) \rightarrow \min _{x \in X} G_{e}(I(x), I(v))=0 .
$$

It follows from Lemma 3.3 (ii) that we may assume $G_{e}\left(I\left(x_{n}\right), I(v)\right)=\varepsilon_{n} \geq 0$ and $\varepsilon_{n} \rightarrow 0$, which implies $I(v) \subset \varepsilon_{n} e+I\left(x_{n}\right)+C$. Then, $\left\{x_{n}\right\}$ is a $k_{0}$-minimizing sequence to $v$. Since $(X, I)$ is $k_{0}$-well-posed at $v$, we have $x_{n} \rightarrow v$.

Now we show that $v$ is the unique minimizer of the scalar problem $\left(X, G_{e}(I(\cdot), I(v))\right.$. In fact, if there exists $u \neq v$ such that $G_{e}(I(u), I(v))=0$, we take $x_{n}=u$ for all $n$. Then, it follows from Lemma 3.3 (i) that $\left\{x_{n}\right\}$ is a $k_{0}$-minimizing sequence to $v$. So $x_{n} \rightarrow v$, which is a contradiction. Thus, $\left(X, G_{e}(I(\cdot), I(v))\right.$ is Tykhonov well-posed.

Conversely, we assume that $\left\{x_{n}\right\}$ is a sequence such that there exists $\varepsilon_{n}>0$ with $\varepsilon_{n} \rightarrow 0$ satisfying $I\left(x_{n}\right) \leq_{C} I(v)+\varepsilon_{n} k_{0}$. Then

$$
0=\min _{x \in X} G_{e}(I(x), I(v)) \leq G_{e}\left(I\left(x_{n}\right), I(v)\right) \leq G_{e}\left(I(v)+\varepsilon_{n} k_{0}, I(v)\right)=\varepsilon_{n} .
$$

Since the scalar problem $\left(X, G_{e}(I(\cdot), I(v))\right)$ is well-posed, $\left\{x_{n}\right\}$ converges to $v$, i.e., the problem $(X, I)$ is $k_{0}$-well-posed at $v$.

(ii) Assume that $\left\{x_{n}\right\}$ is a sequence satisfying

$$
\inf _{v \in \operatorname{argmin}(X, I)} G_{e}\left(I\left(x_{n}\right), I(v)\right) \rightarrow \inf _{x \in X} \inf _{v \in \operatorname{argmin}(X, I)} G_{e}(I(x), I(v))=0 .
$$

Then there exist $\varepsilon_{n}^{\prime}>0, \varepsilon_{n}^{\prime} \rightarrow 0$ and $v_{x_{n}} \in \operatorname{argmin}(X, I)$ such that

$$
0 \leq G_{e}\left(I\left(x_{n}\right), I\left(v_{x_{n}}\right)\right)=\min \left\{r \mid I\left(v_{x_{n}}\right) \subset r e+I\left(x_{n}\right)+C\right\}<\varepsilon_{n}^{\prime} .
$$

It follows from Lemma 3.3 (ii) that there exists $\varepsilon_{n} \in\left[0, \varepsilon_{n}^{\prime}\right)$ satisfying $G_{e}\left(I\left(x_{n}\right), I\left(v_{x_{n}}\right)\right)=$ $\varepsilon_{n}$, which implies $I\left(v_{x_{n}}\right) \subset \varepsilon_{n} e+I\left(x_{n}\right)+C$. Since the problem $(X, I)$ is generalized $k_{0^{-}}$ well-posed, $\left\{x_{n}\right\}$ has a subsequence converging to some point $u \in \operatorname{argmin}(X, I)$. Thus, the scalar optimization problem $\left(X, \inf _{v \in \operatorname{argmin}(X, I)} G_{e}(I(\cdot), I(v))\right)$ is generalized well-posed in the scalar sense.

Conversely, assume that $\left\{x_{n}\right\}$ is a sequence, which satisfies that $\exists \varepsilon_{n}>0, \varepsilon_{n} \rightarrow 0$ and $v_{n} \in \operatorname{argmin}(X, I)$ such that

$$
I\left(x_{n}\right) \leq_{C} I\left(v_{n}\right)+\varepsilon_{n} k_{0} .
$$

Then, from Lemma 3.4 (iii), we have

$$
0=\inf _{x \in X} \inf _{v \in \operatorname{argmin}(X, I)} G_{e}(I(x), I(v))
$$




$$
\begin{aligned}
& \leq \inf _{v \in \operatorname{argmin}(X, I)} G_{e}\left(I\left(x_{n}\right), I(v)\right) \\
& \leq G_{e}\left(I\left(x_{n}\right), I\left(v_{n}\right)\right) \\
& \leq G_{e}\left(I\left(v_{n}\right)+\varepsilon_{n} k_{0}, I\left(v_{n}\right)\right) \\
& =\varepsilon_{n} .
\end{aligned}
$$

Since $\inf _{v \in \operatorname{argmin}(X, I)} G_{e}(I(\cdot), I(v))$ is generalized well-posed in the scalar sense, $\left\{x_{n}\right\}$ has some subsequence converging to some point $u \in \operatorname{argmin}(X, I)$.

(iii) Firstly, we assume that $\left\{x_{n}\right\}$ is a sequence, which satisfies $I\left(x_{n}\right) \not \subset I(x)+\varepsilon_{n} k_{0}+$ $\operatorname{int} C$, for all $x \in X$. Then, from Lemma 3.4 (iv), we have

$$
G_{e}\left(I(x), I\left(x_{n}\right)\right) \geq-\varepsilon_{n}, \forall x \in X
$$

i.e.

$$
-\inf _{x \in X} G_{e}\left(I(x), I\left(x_{n}\right)\right) \leq \varepsilon_{n}
$$

If there exists a subsequence $\left\{x_{n_{k}}\right\} \subset\left\{x_{n}\right\}$ such that $-\inf _{x \in X} G_{e}\left(I(x), I\left(x_{n_{k}}\right)\right)<0$, then

$$
\forall x \in X, \quad G_{e}\left(I(x), I\left(x_{n_{k}}\right)\right)>0
$$

which means that

$$
I\left(x_{n_{k}}\right) \not \subset I(x)+\operatorname{int} C, \quad \forall x \in X .
$$

Thus, $x_{n_{k}}, k=1,2, \cdots$, are the weak minimizers of $(X, I)$. From the compactness of $\operatorname{argwmin}(X, I)$, there exists a subsequence of $\left\{x_{n_{k}}\right\}$ converging to a point in $\operatorname{argwmin}(X, I)$.

If there exists no subsequence $\left\{x_{n_{k}}\right\} \subset\left\{x_{n}\right\}$ such that

$$
-\inf _{x \in X} G_{e}\left(I(x), I\left(x_{n_{k}}\right)\right)<0,
$$

then, there is a subsequence $\left\{x_{n_{k}}^{\prime}\right\} \subset\left\{x_{n}\right\}$ such that

$$
0 \leq-\inf _{x \in X} G_{e}\left(I(x), I\left(x_{n_{k}}^{\prime}\right)\right) \leq \varepsilon_{n_{k}} .
$$

Since for all $v \in \operatorname{argwmin}(X, I), \inf _{x \in X} G_{e}(I(x), I(v))=0$ and $\left(X,-\inf _{x \in X} G_{e}(I(x), I(\cdot))\right)$ is well-posed, there exists a subsequence of $\left\{x_{n_{k}}^{\prime}\right\}$ converging to a point of $\operatorname{argwmin}(X, I)$. Therefore, the problem $(X, I)$ is extended $k_{0}$-well-posed.

Conversely, suppose that $\left\{x_{n}\right\}$ is a sequence, which satisfies that $\exists \varepsilon_{n} \geq 0$ with $\varepsilon_{n} \rightarrow 0$ such that

$$
-\inf _{x \in X} G_{e}\left(I(x), I\left(x_{n}\right)\right) \leq \inf _{v \in X}\left[-\inf _{x \in X} G_{e}(I(x), I(v))\right]+2 \varepsilon_{n}
$$


So for all $v \in X$, we have

$$
\inf _{x \in X} G_{e}\left(I(x), I\left(x_{n}\right)\right) \geq \inf _{x \in X} G_{e}(I(x), I(v))-2 \varepsilon_{n} .
$$

Then, for any $v \in X$ and $n$, there exists a point $y_{n, v} \in X$ such that

$$
G_{e}\left(I(x), I\left(x_{n}\right)\right) \geq G_{e}\left(I\left(y_{n, v}\right), I(v)\right)-\varepsilon_{n}, \forall x \in X .
$$

Especially, taking $x=y_{n, v}$, we have

$$
G_{e}\left(I\left(y_{n, v}\right), I\left(x_{n}\right)\right) \geq G_{e}\left(I\left(y_{n, v}\right), I(v)\right)-\varepsilon_{n} .
$$

It follows from Lemma 3.3 (ii) and Lemma 3.4 (ii) that

$$
I\left(x_{n}\right) \not \subset I(v)+\varepsilon_{n} k_{0}+\operatorname{int} C .
$$

By the arbitrariness of $v$ and $n$, we get

$$
I\left(x_{n}\right) \not \subset I(v)+\varepsilon_{n} k_{0}+\operatorname{int} C, \forall v \in X \text { and } n .
$$

Since $(X, I)$ is extended $k_{0}$-well-posed, there exists a subsequence of $\left\{x_{n}\right\}$ converging to a point $x_{0} \in \operatorname{argwmin}(X, I)$.

Now we show $x_{0} \in \operatorname{argmin}\left(X,-\inf _{x \in X} G_{e}(I(x), I(\cdot))\right)$. In fact, from $x_{0} \in \operatorname{argwmin}(X, I)$, we have that for every $x \in X, G_{e}\left(I(x), I\left(x_{0}\right)\right) \geq 0$. Arbitrarily choosing $y \in X$, we obtain

$$
\inf _{x \in X} G_{e}\left(I(x), I\left(x_{0}\right)\right) \geq G_{e}(I(y), I(y))
$$

by Lemma 3.3 (iii). Then, for every $y \in X$,

$$
-\inf _{x \in X} G_{e}(I(x), I(y)) \geq-\inf _{x \in X} G_{e}\left(I(x), I\left(x_{0}\right)\right),
$$

i.e., $x_{0} \in \operatorname{argmin}\left(X,-\inf _{x \in X} G_{e}(I(x), I(\cdot))\right)$. Thus, the scalar problem $\left(X,-\inf _{x \in X}\right.$ $\left.G_{e}(I(x), I(\cdot))\right)$ is generalized well-posed in the scalar sense. This completes the proof.

Corollary 3.1 Let $X$ be a compact space, $I$ be a $C$-bounded and $C$-lower semi-continuous mapping defined on $X$. Suppose that for every $v \in \operatorname{argmin}(X, I)$, there exists no point $u \in X, u \neq v$ satisfying both $I(v) \leq_{C} I(u)$ and $I(u) \leq_{C} I(v)$. Then, $(X, I)$ is $k_{0}$-wellposed at $v$. 
Proof. Naturally, for any real number $\alpha$,

$$
\left\{x \in X: G_{e}(I(x), I(v)) \leq \alpha\right\}=\{x \in X: I(v) \subset \alpha e+I(x)+C\} .
$$

From the closedness of the set $\{x \in X: A \subset I(x)+C, \forall A \subset Y\}$, we have that the set

$$
\left\{x \in X: G_{e}(I(x), I(v)) \leq \alpha\right\}
$$

is also closed for any $\alpha$. So, $G_{e}(I(\cdot), I(v))$ is lower semi-continuous on $X$. From the compactness of $X$ and Example 6 of [19, p.3], the scalar problem $\left(X, G_{e}(I(\cdot), I(v))\right)$ is Tykhonov well-posed. Thus, by Theorem 3.1 (i), we get that $(X, I)$ is $k_{0}$-well-posed at $v$.

Corollary 3.2 Let $X$ be a locally compact metric space. Assume that $v \in \operatorname{argmin}(X, I), I$ is $C$-bounded-valued and $C$-closed-valued and $\left\{x \in X: \forall \varepsilon>0, \exists v \in \operatorname{argmin}(X, I), I(x) \leq_{C}\right.$ $\left.I(v)+(t+\varepsilon) k_{0}\right\}$ is connected. Then, the following three assertions are equivalent:

(i) $\exists t>0,\left\{x \in X: \forall \varepsilon>0, \exists v \in \operatorname{argmin}(X, I), I(x) \leq_{C} I(v)+(t+\varepsilon) k_{0}\right\}$ is compact;

(ii) $(X, I)$ is generalized $k_{0}$-well-posed;

(iii) $\operatorname{argmin}(X, I)$ is nonempty and compact.

Proof. We know the set $\left\{x \in X: \inf _{v \in \operatorname{argmin}(X, I)} G_{e}(I(x), I(v)) \leq t\right\}$ is equivalent to the set $\left\{x \in X: \forall \varepsilon>0, \exists v \in \operatorname{argmin}(X, I), G_{e}(I(x), I(v)) \leq t+\varepsilon\right\}$, which is also equivalent to the set $\left\{x \in X: \forall \varepsilon>0, \exists v \in \operatorname{argmin}(X, I), I(x) \leq_{C} I(v)+(t+\varepsilon) k_{0}\right\}$. So, assumption (i) is equivalent to the conclusion that

$$
\left\{x \in X: \inf _{v \in \operatorname{argmin}(X, I)} G_{e}(I(x), I(v)) \leq t\right\}
$$

is compact for some $t>\inf _{x \in X} \inf _{v \in \operatorname{argmin}(X, I)} G_{e}(I(x), I(v))=0$. From Proposition 37 of [19, p.25] and Theorem 3.1 (ii), we conclude that (i), (ii) and (iii) are equivalent.

Remark 3.1 When $I$ is scalar-valued, $C=R_{+}$and $k_{0}=1$, Corollary 3.1 reduces to Example 6 of [19, p.3] and Corollary 3.2 reduces to Proposition 37 of [19, p.25].

As [19], we introduce a function $c: R_{+} \rightarrow R$, which is called a generalized forcing function if and only if $c(t) \geq 0, c(0)=0$ and

$$
t_{n} \geq 0, c\left(t_{n}\right) \rightarrow 0 \Rightarrow \exists\left\{t_{n_{k}}\right\} \subset\left\{t_{n}\right\} \text { such that } t_{n_{k}} \rightarrow 0 \quad(k \rightarrow \infty) .
$$


Theorem 3.2 Assume that $X$ is a metric space and $I$ is $C$-bounded-valued and $C$-closedvalued. Then,

(i) if $(X, I)$ is generalized $k_{0}$-well-posed, for any fixed $x \in X$, there exists some generalized forcing function $c$ satisfying, for all $x^{\prime} \in \operatorname{argmin}(X, I)$,

$$
I\left(x^{\prime}\right) \not \subset I(x)+c(d(x, \operatorname{argmin}(X, I)) e+\operatorname{int} C .
$$

Conversely, if $\operatorname{argmin}(X, I) \neq \emptyset$ and $\operatorname{argmin}(X, I)$ is compact, (6) holds for some $c$ satisfying (5), then $(X, I)$ is generalized $k_{0}$-well-posed;

(ii) $(X, I)$ is $k_{0}$-well-posed at $v \in \operatorname{argmin}(X, I)$ if and only if there exists a generalized forcing function c satisfying $I(v) \not \subset I(x)+c(d(x, v)) e+i n t C$.

Proof. (i) Let $(X, I)$ be generalized $k_{0}$-well-posed. Define

$$
c(t)=\inf \left\{G_{e}\left(I(x), I\left(x^{\prime}\right)\right): d(x, \operatorname{argmin}(X, I))=t, x^{\prime} \in \operatorname{argmin}(X, I)\right\} .
$$

It is easy to see that $c(t) \geq 0$. We conclude that $c(0)=0 \operatorname{since} \operatorname{argmin}(X, I)$ is compact.

Now let $t_{n} \geq 0$ with $c\left(t_{n}\right) \rightarrow 0$. Then $\exists x_{n} \in X, x_{n}^{\prime} \in \operatorname{argmin}(X, I), \varepsilon_{n}>0$ with $\varepsilon_{n} \rightarrow 0$ such that

$$
d\left(x_{n}, \operatorname{argmin}(X, I)\right)=t_{n}
$$

and

$$
G_{e}\left(I\left(x_{n}\right), I\left(x_{n}^{\prime}\right)\right) \leq \varepsilon_{n}
$$

Thus, we have

$$
I\left(x_{n}^{\prime}\right) \subset \varepsilon_{n} e+I\left(x_{n}\right)+C .
$$

From the definition of generalized $k_{0}$-well-posedness, we have that there exists a subsequence $\left\{x_{n_{k}}\right\}$ such that $d\left(x_{n_{k}}, \operatorname{argmin}(X, I)\right) \rightarrow 0$, namely, there exists a subsequence $\left\{t_{n_{k}}\right\}$ of $\left\{t_{n}\right\}$ such that $t_{n_{k}} \rightarrow 0$.

In addition, by the definition of $c(t)$, we have $\forall x^{\prime} \in \operatorname{argmin}(X, I)$, such that

$$
G_{e}\left(I(x), I\left(x^{\prime}\right)\right) \geq c(d(x, \operatorname{argmin}(X, I)),
$$

which implies

$$
I\left(x^{\prime}\right) \not \subset I(x)+c(d(x, \operatorname{argmin}(X, I)) e+\operatorname{int} C .
$$

Conversely, if for $x_{n} \in X, \varepsilon_{n}>0$ with $\varepsilon_{n} \rightarrow 0, \exists x_{n}^{\prime} \in \operatorname{argmin}(X, I)$, such that

$$
I\left(x_{n}^{\prime}\right) \subset I\left(x_{n}\right)+\varepsilon_{n} e+C,
$$


then we have

$$
\varepsilon_{n} \geq G_{e}\left(I\left(x_{n}\right), I\left(x_{n}^{\prime}\right)\right) \geq c\left(d\left(x_{n}, \operatorname{argmin}(X, I)\right) .\right.
$$

So,

$$
d\left(x_{n}, \operatorname{argmin}(X, I)\right) \rightarrow 0 .
$$

From the compactness of $\operatorname{argmin}(X, I)$, we conclude that $(X, I)$ is generalized $k_{0}$-wellposed.

(ii) The proof of (ii) is similar to that of (i). So we omit it.

\section{Criteria and Characterizations of Well-Posedness}

Now we consider some characterizations and criteria of well-posedness for set optimization problems. For every bounded set $A \subset X$, we recall the Kuratowski measure of noncompactness of $A$ (see [2]):

$$
\alpha(A)=\inf \{k>0: \text { A has a finite cover of sets with diameter }<k\} .
$$

The generalized $k_{0}$-well-posedness (extended $k_{0}$-well-posedness) can be characterized by the behavior of $\varepsilon-\operatorname{argmin}(X, I)\left(\varepsilon-\operatorname{argmin}^{\prime}(X, I)\right)$ as $\varepsilon \rightarrow 0$, which is defined as

$$
\begin{gathered}
\varepsilon-\operatorname{argmin}(X, I)=\left\{x \in X \mid I(z)+\varepsilon k_{0} \subset I(x)+C, \exists z \in \operatorname{argmin}(X, I)\right\} \\
\left(\varepsilon-\operatorname{argmin}^{\prime}(X, I)=\left\{x \in X \mid I(z)+\varepsilon k_{0} \not_{\text {int } C} I(x), \forall z \in X\right\}\right) .
\end{gathered}
$$

It is clear that $\varepsilon-\operatorname{argmin}(X, I) \neq \emptyset$, and if $I(X)$ is $C$-bounded, then $\varepsilon-\operatorname{argmin}^{\prime}(X, I) \neq \emptyset$.

Proposition 4.1 Suppose that $I: X \rightarrow 2^{Y}$ is compact-valued and $\varepsilon-\operatorname{argmin}(X, I)$ is closed. Then the problem $(X, I)$ is generalized $k_{0}$-well-posed (extended $k_{0}$-well-posed) and $\operatorname{argmin}(X, I)\left(\operatorname{argmin}^{\prime}(X, I)\right)$ is compact if and only if

$$
\alpha[\varepsilon-\operatorname{argmin}(X, I)] \rightarrow 0\left(\alpha\left[\varepsilon-\operatorname{argmin}^{\prime}(X, I)\right] \rightarrow 0\right)
$$

as $\varepsilon \rightarrow 0$.

Proof. Put

$$
L(\varepsilon)=\varepsilon-\operatorname{argmin}(X, I) .
$$

Similar to Theorem 3.2 in [2], we only need to verify $\operatorname{argmin}(X, I)=\cap_{\varepsilon>0} L(\varepsilon)$. 
Let $x_{1} \in \bigcap_{\varepsilon>0} L(\varepsilon)$ and take any $\varepsilon>0$. Then there exists $z_{0} \in \operatorname{argmin}(X, I)$ such that

$$
I\left(z_{0}\right)+\varepsilon k_{0} \subset I\left(x_{1}\right)+C .
$$

Let $\varepsilon \rightarrow 0$. Since $I$ is compact-valued, we have

$$
I\left(z_{0}\right) \subset I\left(x_{1}\right)+C
$$

By virtue of $z_{0} \in \operatorname{argmin}(X, I)$, we deduce that the point $x_{1}$ must be in $\operatorname{argmin}(X, I)$. Thus, $\bigcap_{\varepsilon>0} L(\varepsilon) \subset \operatorname{argmin}(X, I)$. On the other hand, it is clear that

$$
\operatorname{argmin}(X, I) \subset \bigcap_{\varepsilon>0} L(\varepsilon) .
$$

So, we have proved that $\operatorname{argmin}(X, I)=\bigcap_{\varepsilon>0} L(\varepsilon)$.

Similarly, let $L^{\prime}(\varepsilon)=\varepsilon-\operatorname{argmin}^{\prime}(X, I)$. We only need to verify

$$
\operatorname{argwmin}(X, I)=\cap_{\varepsilon>0} L^{\prime}(\varepsilon) .
$$

The inclusion relation $\operatorname{argwmin}(X, I) \subset \cap_{\varepsilon>0} L^{\prime}(\varepsilon)$ holds obviously. Now we show that $\cap_{\varepsilon>0} L^{\prime}(\varepsilon) \subset \operatorname{argwmin}(X, I)$. In fact, if $x \in \cap_{\varepsilon>0} L^{\prime}(\varepsilon)$ but $x \notin \operatorname{argwmin}(X, I)$, then there exist $z_{0} \in X, \varepsilon_{n} \rightarrow 0^{+}$and $y_{n} \in I(x)$ such that

$$
I(x) \subset I\left(z_{0}\right)+\operatorname{int} C
$$

and

$$
y_{n} \notin I\left(z_{0}\right)+\varepsilon_{n} k_{0}+\operatorname{int} C .
$$

Since $I$ is compact-valued, without loss of generality, we may assume that $y_{n} \rightarrow y_{0} \in I(x)$. So, $y_{0} \notin I\left(z_{0}\right)+\operatorname{int} C$, which contradicts $(7)$. The proof is complete.

Let

$$
L(v, \alpha):=\left\{x \in X \mid I(x) \leq_{C} I(v)+\alpha k_{0}\right\} .
$$

Proposition 4.2 Assume $I: X \rightarrow 2^{Y}$ is compact-valued. Then, the set optimization problem $(X, I)$ is $k_{0}$-well-posed at $v \in \operatorname{argmin}(X, I)$ if and only if $\inf _{\alpha} \operatorname{diam} L(v, \alpha)=0$.

Proof. The proof is similar to that of Theorem $11[19$, p.5]. So it is omitted.

Let $X^{*}$ be the topological dual space of $X$ and $C^{*}=\left\{b \in X^{*} \mid b(c) \geq 0, \forall c \in C\right\}$. We define the function $I_{\varepsilon}=I+\varepsilon d(\cdot, v) k_{0}$, where $v \in \operatorname{argmin}(X, I)$ and $\varepsilon>0$. Obviously, $v \in \operatorname{argmin}\left(X, I_{\varepsilon}\right)$. 
Theorem 4.1 Assume that $I: X \rightarrow 2^{Y}$ is convex-valued, compact-valued and bounded valued. Then the set optimization problem $\left(X, I_{\varepsilon}\right)$ is $k_{0}$-well-posed at $v \in \operatorname{argmin}(X, I)$.

Proof. From Proposition 4.2, we only need to prove that there exists a constant $d$, which is independent of $\alpha$, such that

$$
L_{\varepsilon}(v, \alpha):=\left\{x \in X \mid I_{\varepsilon}(x) \leq_{C} I(v)+\alpha k_{0}\right\} \subset B(v, \alpha d),
$$

where $B(v, \alpha d)$ is a ball at $v$ with radius $\alpha d$. Let $x \in L(v, \alpha)$ with $x \neq v$.

If $I(x) \mathbb{L}_{C} I(v)$, then there exists $y \in I(v)$ such that $y \cap(I(x)+C)=\emptyset$. Especially, we have $y \cap I(x)=\emptyset$. Since $I$ is convex valued, there exists an $b \in C^{*}$ such that for all $y_{1} \in I(x)$,

$$
b(y)<b\left(y_{1}\right)
$$

Then,

$$
\min b(I(v))<\min b(I(x)) .
$$

Let $\bar{b} \in C^{*}$ satisfying $\bar{b}\left(k_{0}\right)>0$. Since $I$ is compact-valued, we may choose an $\beta>0$ such that

$$
\min (b+\beta \bar{b})(I(v))<\min (b+\beta \bar{b})(I(x)) .
$$

It follows from $x \in L_{\varepsilon}(v, \alpha)$ that

$$
\begin{aligned}
\min (b+\beta \bar{b})\left(I(x)+\varepsilon d(x, v) k_{0}\right) & \leq \min (b+\beta \bar{b})\left(I(v)+\varepsilon d(v, v) k_{0}\right)+\alpha(b+\beta \bar{b})\left(k_{0}\right) \\
& <\min (b+\beta \bar{b})(I(x))+\alpha(b+\beta \bar{b})\left(k_{0}\right) .
\end{aligned}
$$

Hence, $\varepsilon d(x, v)(b+\beta \bar{b})\left(k_{0}\right)<\alpha(b+\beta \bar{b})\left(k_{0}\right)$, i.e.

$$
d(x, v) \leq \alpha / \varepsilon .
$$

If $I(x) \leq_{C} I(v)$, then $I(v) \leq_{C} I(x)$. Since $x \in L(v, \alpha)$, we conclude that

$$
I(v)+\varepsilon d(x, v) k_{0} \leq_{C} I(x)+\varepsilon d(x, v) k_{0}=I_{\varepsilon}(x) \leq_{C} I(v)+\alpha k_{0},
$$

i.e.,

$$
I(v)+\alpha k_{0} \subset I(v)+\varepsilon d(x, v) k_{0}+C .
$$

If $\alpha<\varepsilon d(x, v)$, then,

$$
I(v) \subset I(v)+(\varepsilon d(x, v)-\alpha) k_{0}+C \subset I(v)+\operatorname{int} C,
$$


which is a contradiction. So, we have that $\varepsilon d(x, v) \leq \alpha$, i.e., $d(x, v) \leq \alpha / \varepsilon$. Let $d=1 / \varepsilon$. We conclude that $d(x, v) \leq \alpha d$, for each $x \in L(v, \alpha)$. It is said that $L(v, \alpha) \subset B(v, \alpha d)$.

For any $k_{0} \in \operatorname{int} C$, let

$$
\begin{gathered}
M(\varepsilon)=\varepsilon-\operatorname{argmin}(X, I), \\
M^{\prime}(\varepsilon, v)=\left\{x \in X \mid I(v)+\varepsilon k_{0} \subset I(x)+C\right\}, \\
M^{\prime \prime}(\varepsilon)=\varepsilon-\operatorname{argmin}^{\prime}(X, I) .
\end{gathered}
$$

Proposition 4.3 (i) $M(\cdot)$ is u.s.c. at 0 and $\operatorname{argmin}(X, I)$ is compact iff the problem $(X, I)$ is generalized $k_{0}$-well-posed.

(ii) $M^{\prime}(\cdot, v)$ is u.s.c. at 0 and $M^{\prime}(0, v)=\{v\}$ iff the problem $(X, I)$ is $k_{0}$-well-posed at $v$.

(iii) $M^{\prime \prime}(\cdot)$ is u.s.c. at 0 and $\operatorname{argwmin}(X, I) \neq \emptyset$ is compact iff the problem $(X, I)$ is extended $k_{0}$-well-posed.

Proof. We only prove that (i) holds, since the proofs of (ii) and (iii) are similar to that of (i).

First of all, it is easy to prove that

$$
M(0)=\operatorname{argmin}(X, I) .
$$

Suppose that $(X, I)$ is generalized $k_{0}$-well-posed. It follows from Remark 2.1 that $\operatorname{argmin}(X, I)$ is compact. So, we only need to prove that $M(\cdot)$ is u.s.c. at 0 . Suppose $M(\cdot)$ is not u.s.c.. Then there is a neighborhood $N(M(0))$ of $M(0)$, such that for any neighborhood $U$ of 0 , there exists $\varepsilon^{\prime}$ satisfying

$$
\left\{x \in X \mid I(z)+\varepsilon^{\prime} k_{0} \subset I(x)+C, \exists z \in \operatorname{argmin}(X, I)\right\} \not \subset N(M(0)) .
$$

Thus, we can choose $\varepsilon_{n} \rightarrow 0$ satisfying

$$
\exists x_{n} \in X, z_{n} \in \operatorname{argmin}(X, I),
$$

such that

$$
I\left(z_{n}\right)+\varepsilon_{n} k_{0} \subset I\left(x_{n}\right)+C \text { and } x_{n} \notin N(M(0)) .
$$

We deduce from (8) and (9) that $\left\{x_{n}\right\}$ is a generalized $k_{0}$-minimizing sequence. Therefore, there exist a subsequence $\left\{x_{n_{k}}\right\}$ and $z \in \operatorname{argmin}(X, I)$ such that $x_{n_{k}} \rightarrow z$. From (9), $x_{n_{k}} \notin N(M(0))$, which is a contradiction. 
Conversely, suppose that $\exists \varepsilon_{n} \geq 0$ with $\varepsilon_{n} \rightarrow 0, x_{n} \in X, z_{n} \in \operatorname{argmin}(X, I)$ such that

$$
I\left(z_{n}\right)+\varepsilon_{n} k_{0} \subset I\left(x_{n}\right)+C .
$$

Since $M(\cdot)$ is u.s.c. at 0 , for any $N(M(0))$, when $n$ is sufficiently large, we get that $x_{n} \in N(M(0))$. Therefore, for every neighborhood $W$ of 0 , there exists $n_{0} \in N$ such that $x_{n} \in \operatorname{argmin}(X, I)+W, \forall n>n_{0}$. By the compactness of $\operatorname{argmin}(X, I)$, we obtain that the problem $(X, I)$ is generalized $k_{0}$-well-posed.

\section{References}

[1] A. N. Tykhonov, On the stability of the functional optimization problem, USSR Compt. Math. Math. Phys., 6 (1966) 28-33.

[2] X. X. Huang, Extended and strongly extended well-posedness of set-valued optimization problems, Math. Methods Oper. Res., 53 (2001) 101-116.

[3] X. X. Huang, Pointwise well-posedness of perturbed vector optimization problems in a vector-valued variational principle, J. Optim. Theory Appl., 108(3) (2001) 671-686.

[4] E. Miglierina, E. Molho, M. Rocca, Well-posedness and scalarization in vector optimization, J. Optim. Theory Appl., 126(2) (2005) 391-409.

[5] D. Dentcheva, S. Helbig, On variational principles, level sets, well-posedness and $\varepsilon$-solutions in vector optimization, J. Optim. Theory Appl., 89 (1996) 329-349.

[6] R. Hu, Y. P. Fang, Set-valued increasing-along-rays maps and well-posed set-valued star-shaped optimization, J. Math. Anal. Appl., 331 (2007) 1371-1383.

[7] M. B. Lignola, Well-posedness and L-well-posedness for quasivariational inequalities, J. Optim. Theory Appl., 128(1) (2006) 119-138.

[8] R. Lucchetti, I. Revalski, (Eds.) Recent development in well-posed variational problems, Kluwer Academic Publishers, Dordrecht, 1995.

[9] E. M. Bednarczuk, An approach to well-posedness in vector optimization problems: consequences to stability, Control and Cybernetics, 23(1994) 107-122.

[10] M. Rocca, Well-posed vector optimization problems and vector variational inequalities. J. Inf. Optim. Sci., 27(2) (2006) 259-270. 
[11] X. X. Huang, X. Q. Yang, Levitin-Polyak well-posedness in generalized variational inequality problems with functional constraints, J. Ind. Manag. Optim., 3(4) (2007) 671-684.

[12] D. Kuroiwa, Some duality theorems of set-valued optimization with natural criteria, in: Proceedings of the International Conference on Nonlinear Analysis and Convex Analysis, World Scientific, River Edge, NJ, (1999) 221-228.

[13] D. Kuroiwa, On set-valued optimization, Nonlinear Anal., 47 (2001) 1395-1400.

[14] D. Kuroiwa, Existence theorems of set optimization with set-valued maps, J. Inf. Optim. Sci., 24 (2003) 73-84.

[15] D. Kuroiwa, Existence of efficient points of set optimization with weighted criteria, J. Nonlinear Convex Anal., 4 (2003) 117-123.

[16] T. X. D. Ha, Some variants of Ekeland variational priciple for a set-valued map, J. Optim. Theory Appl., 124(1) (2005) 187-206.

[17] E. Hernández, L. Rodríguez-Marín, Nonconvex scalarization in set optimization with set-valued maps, J. Math. Anal. Appl., 325(1) (2007) 1-18.

[18] M. Alonso, L. Rodríguez-Marín, Set-relations and optimality conditions in set-valued maps, Nonlinear Anal., 63 (2005) 1167-1179.

[19] A. L. Dontchev, T. Zolezzi, Well-posed optimization Problems. In: Lecture Notes in Mathematics, 1543, Springer-Verlag, Berlin, 1993.

[20] A. Göpfort, H. Riahi, C. Tammer, C. Zălinescu, Variational methods in partially ordered spaces, Springer, New York, 2003. 